\section{Subversion of MAPK signaling by pathogenic bacteria}

\author{
Lihi Gur-Arie, Ilan Rosenshine \\ Department of Microbiology and \\ Molecular Genetics, Institute of Medical \\ Research Israel-Canada, Faculty of \\ Medicine, The Hebrew University of \\ Jerusalem, Israel
}

\begin{abstract}
Bacterial components are recognized by host pattern recognition receptors that trigger signaling cascades, leading to inflammation and eradication of the bacteria. The main proinflammatory signaling pathway is the MAP kinase (MAPK)/NF- $\kappa \mathrm{B}$ interwoven cascades, which result in transcription of pro-inflammatory genes. Many bacteria have evolved to interfere with the immune response through a mechanism that involves delivery of virulent proteins to the host cells. These proteins posttranslationally modify key components in the host signaling cascades. This review will describe bacterial strategies to directly manipulate host MAPK signaling, summarizing recent discoveries in the field.
\end{abstract}

\section{Introduction}

The innate immune response provides the first line of defense against a microbial attack. Upon infection, bacterial components, termed pathogen-associated molecular patterns (PAMPs) and the necrotic cell content are recognized by the host pattern recognition receptors. PAMP recognition elicits activation of the MAP3K TAK1, which activate the MAPK/IKK/NF- $\kappa$ B signaling pathways. 1,2 These pathways lead to transcription of multiple genes that control the immune response, including the pro-inflammatory cytokines interleukin-1 $\beta$ (IL-1 $\beta$ ) and IL-8, resulting in recruitment to the site of infection of phagocytic cells and other components of the immune response. ${ }^{3}$

To survive and sustain a stable infection, the pathogen must cope with the host immune response. This is often achieved by delivering into the host cells virulence factors that subvert the host inflammatory response and associated signaling pathways. Delivery of these factors is attained by secretion of exotoxins to the extracellular milieu, or via direct injection of proteins, termed effectors, to the host cell by attached bacteria. To inject these effectors bacteria employ surface organelles that function as nano-injectors including type three and type four secretion systems (T3SS and T4SS). In most cases the attached bacteria inject dozens of these effectors. Common targets for these effectors are cellular immune response cascades, including MAPK/NF-кB pathway.,5 Importantly, in many cases pathogens employ several effectors that act sequentially or simultaneously on different components of the same signaling network to obtain the desired outcome. Furthermore, the entire effector cohort acts in concert on a number of signaling pathways to allow efficient bacterial colonization of the host.

This short review is limited to an updated description of bacterial strategies to manipulate host MAPK signaling through translocated effectors or exotoxins that directly catalyze posttranslational modification of proteins of the MAPK network including MAP3Ks, MAP2Ks and MAPKs (Table 1, Figures 1 and 2). ${ }^{6-30}$ Virulence factors interfering with input and output of the MAPK pathways (i.e., host components acting upstream to MAP3K or downstream to MAPK) will be mentioned, but will not be discussed extensively.

\section{Enteropathogenic Escherichia coli and related pathogens tar- get p38 and JNK signaling cas- cades}

Enteropathogenic and enterohemorrhagic Escherichia coli (EPEC and EHEC, respectively) are closely related pathogens that colonize the human small bowel or colon. They are etiological agents of severe watery diarrhea and dysentery. EPEC remain a significant cause of infant mortality, with 79,000 annual deaths. ${ }^{31}$ EHEC induce severe symptoms, causing diarrhea complicated by hemorrhagic colitis and occasionally hemolytic uremic syndrome, which can be fatal. ${ }^{32}$ To facilitate infection, these pathogens translocate effector proteins into host cell via T3SS, some of which manipulate MAPK/NF- $\kappa B$ pathway. Two of these effectors, NleD and NleE, directly act on MAPK signaling components.

NleD is a zinc metalloprotease that specifically cleaves the host JNK and p38 MAPKs, but not ERK. NleD cleaves these MAPKs within the activation loop that connects the MAPK N- and C-terminal. The loop contains conserved threonine and tyrosine separated by glycine or proline (TXY motif) and MAPK activation is mediated by phosphorylation of the threonine and tyrosine residues of this motif. Importantly, NleD cleaves JNK and p38 between the Gly/Pro and Tyr of the TXY motif rendering them permanently inactive. BLAST search identified a plethora of proteins that share homology with NleD. These proteins are found in pathogenic
Correspondence: Ilan Rosenshine, Department of Microbiology and Molecular Genetics, Institute of Medical Research Israel-Canada, Faculty of Medicine, The Hebrew University of Jerusalem, P.0.B. 12272, Ein Kerem, Jerusalem 9112102, Israel.

Tel.: 972.2.6758754. E-mail: ilanr@ekmd.huji.ac.il

Key words: MAPK signaling; pathogenic bacteria; host-pathogen interaction; toxins; effectors.

Acknowledgments: the work in the IR laboratory was supported by a grant from the Israel Science Foundation funded by The Israel Academy of Science and Humanities. IR is an Etta Rosensohn Professor of Bacteriology.

Contributions: the authors contributed equally.

Conflict of interest: the authors declare no potential conflict of interest.

Received for publication: 4 June 2015.

Revision received: 10 August 2015

Accepted for publication: 1 September 2015.

This work is licensed under a Creative Commons Attribution NonCommercial 3.0 License (CC BYNC 3.0).

(C) Copyright L. Gur-Arie and I. Rosenshine, 2015 Licensee PAGEPress, Italy

MAP Kinase 2015; 4:5303

doi:10.4081/mk.2015.5303

bacteria that cause disease in mammals (EPEC, EHEC, Citrobacter rodentium and Salmonella enterica ssp. Arizonae), in plants (Pseudomonas syringae), or in symbiotic bacteria (Hamiltonella defensa). This implies that EPEC NleD is a representative of a family of NleD-like proteins. ${ }^{6}$ These proteins are probably zinc metalloproteases and possibly target MAPKs.

An additional EPEC effector disrupting MAPK signaling is NleE, which inhibits activation of MAP3K TAK1 (transforming growth factor $\beta$ activated kinase-1). TAK1 forms a complex with TAB2/3, a ubiquitin-binding protein that specifically binds to K63-linked ubiquitin chains and likely also to linear-ubiquitin chains. ${ }^{7}$ Binding of TAB2/3 to the ubiquitin chains results in TAK1 activation. NleE is a methyltransferase that specifically methylates a conserved cysteine residue in the Npl4-like Zinc Finger (NZF) domains of TAB2/3. This modification abolishes the TAB $2 / 3$ ability to bind to the ubiquitin chain, preventing TAK1 activation. ${ }^{7}$ Since TAK1 transmits a signal to both the MAPK and NF- $\kappa B$ pathways, both are repressed. This repression prevents production of pro-inflammatory chemokines, such as IL-6, IL-8 and TNF.33-35 NleE and NleD are translocated at the same time to the host cell. ${ }^{36}$ 
It is thus intriguing why EPEC evolved two different effectors that redundantly block JNK and p38 signaling.

\section{Shigella flexneri reprogram the host epigenome through MAPK inactivation}

Shigella flexneri ( $S$. flexneri) is facultative intracellular pathogen that causes shigellosis or bacillary dysentery, and accounts for about one-third of the total annual deaths due to enteric infections. ${ }^{37}$ S. flexneri employs a T3SS to invade the intestinal epithelium cells, causing inflammation and tissue damage. $0 \mathrm{spF}$ is one of the T3SS injected effectors and it targets MAPKs. To recognize MAPKs, OspF mimics the canonical D motif, found in many MAPK substrates, that is required for MAPK docking. OspF is phospho-threonine-lyase that catalyses a $\beta$ elimination reaction to irreversibly remove the phosphate moiety from a phosphor-threonine residue in the TXY motif of MAPKs. ${ }^{8,9}$ This activity irreversibly and permanently inactivates the modified MAPK. Although OspF might dephosphorylate all three families of MAPKs in vitro, ${ }^{8}$ it seems that only ERK and p38 are its native substrates in vivo. 10 Inactivation of ERK and $\mathrm{p} 38 \beta$ by $0 \mathrm{spF}$ promotes chromatin condensation and inhibition of transcriptional activation by masking NF- $\kappa \mathrm{B}$ binding sites, leading to compromised recruitment of polymorphonuclear leukocytes to infected tissues. ${ }^{10}$ It was further reported that inactivation of ERK by $0 \mathrm{spF}$ reduces the activity of the downstream kinase MSK1, thus reducing up-regulation of proliferative genes and fine-tuning of immune gene expression. ${ }^{38}$ $S$. flexneri also possesses an NleE homologue, OspZ, although the gene is not functional in all isolates. Like NleE, OspZ blocks NF- $\kappa$ B activation, 35 probably by TAB2/3 methylation.

\section{The Yersinia effector YopJ inhibits MAPK activation by MAP2K acetylation}

Yersinia strains (Yersinia st.) cause disease ranging from self-limited gastroenteritis (Yersinia enterocolitica and Yersinia pseudotuberculosis) to a devastating life threatening plague (Yersinia pestis). It is a gram negative, rod-shaped, facultative anaerobic bacterium. Yersinia's virulence is dependent on a T3SS and injected effectors, including YopJ (YopP in $Y$. enterocolitica). Initially, YopJ was proposed to act as a deubiquitinating protease, 39,40 but more recent studies indicate that YopJ is an acetyl-transferase that transfers an acetyl group from acetyl-coenzyme A to specific residues of target proteins. YopJ inhibits the MAPK pathway by acetylating serine, threonine and lysine residues in the activation loop of various MAP2Ks, including MKK2, MKK4, MKK6, MKK7 and the upstream MAP3K-TAK1.11-13 Upon acetylation, these host proteins can no longer undergo phosphorylation, thus compromising the respective MAPK signaling pathway. NF- $\mathrm{KB}$ signaling is also attenuated by YopJ that acetylates IKK $\alpha / \beta$ and prevents its activation. By modulating these signaling pathways, YopJ induces apoptosis and inhibits pro-inflammatory signal transduction, preventing the production of protective cytokines such as TNF $\alpha$ and IL- 8.41

\section{Salmonella enterica directly activate and repress MAPKs}

Salmonella enterica ( $S$. enterica) serovar Typhimurium is the etiological agent of gastroenteritis and systemic infections. This pathogen colonizes many niches in the host and alternates between extracellular and intracellular lifestyles. $S$. enterica employs two distinct T3SS, SPI1 and SPI2, to inject into the host a complex array of effectors. Using two T3SS provides $S$. enterica with better control over the temporal and spatial resolution of the repertoire of injected effectors. For example,

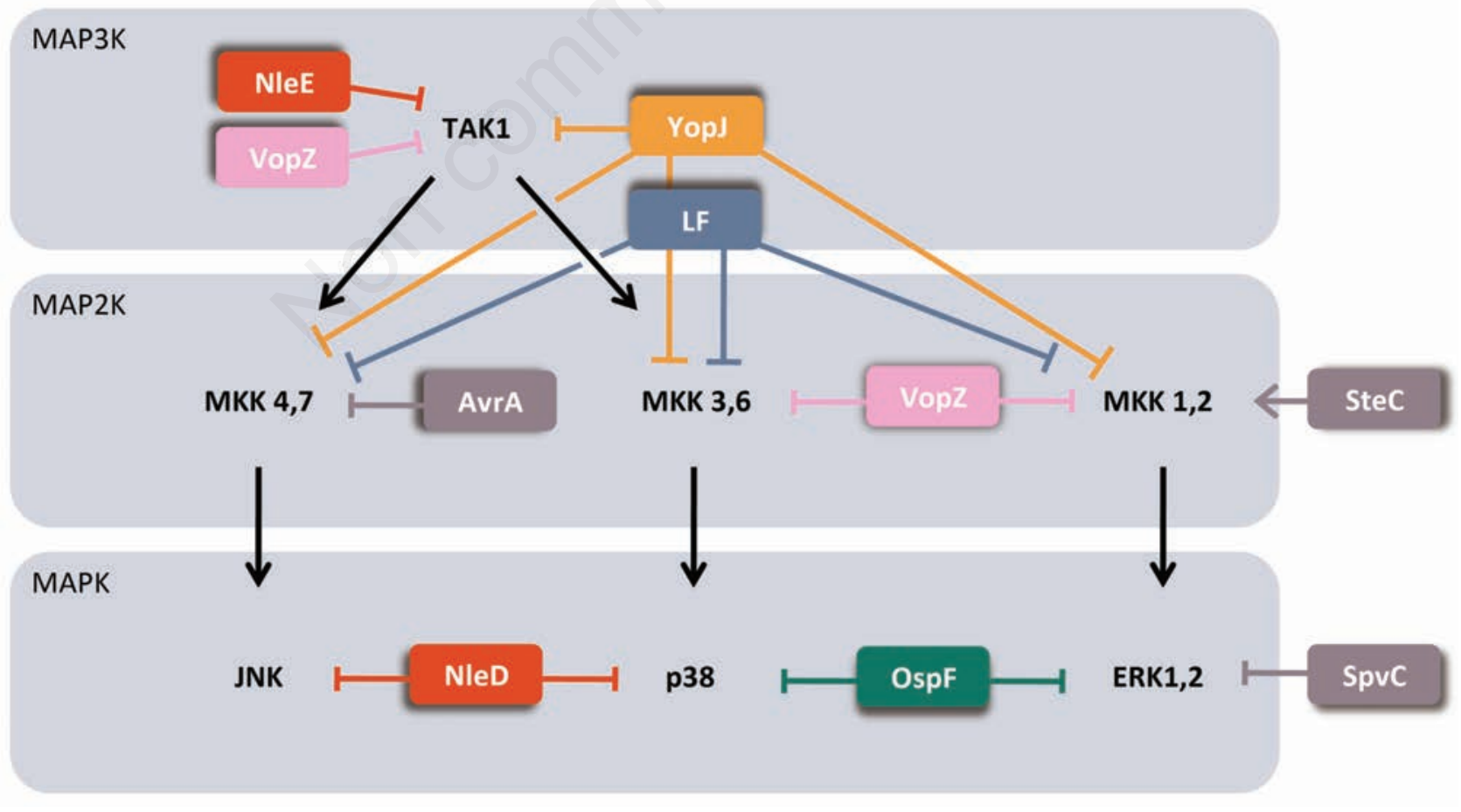

Figure 1. Manipulation of mammalian MAPK signaling by bacterial proteins. MAPK proteins are colored in black, and divided to three tiers; MAPK, MAP2K and MAP3K. Bacterial proteins that interfere with MAPK cascade are marked in squares, and each color represents different bacterial specie; enteropathogenic $E$. coli (red), Yersinia (orange), V. parahaemolyticus (pink), S. enterica (purple), S. flexneri (green), B. anthracis (blue). 
the SPI1 T3SS is used mainly by extracellular bacteria and the SPI 2 by intracellular $S$. enterica. Some $S$. enterica effectors have pro-inflammatory activity, while others reduce the host immune response by inhibiting inflammation signaling. ${ }^{42-44}$

Some $S$. enterica carry NleD and NleE homologues ( $S$. enterica ssp. Arizonae), but these effectors are missing in most $S$. enterica isolates. More common MAPK-manipulating effectors of $S$. enterica are SpvC, AvrA, and SteC. SpvC is very similar to $0 \mathrm{spF}$ of $S$. flexneri and is also a phospho-threoninelyase of MAPKs. Upon over expression, or in vitro, SpvC can target ERK1/2, p38 and JNK,, 14 but in vivo, under physiological conditions, only ERK1/2 are dephosphorylated. 15 Genetic analyses show that $\mathrm{SpvC}$ is required for systemic infection in mice. 45 Inhibition of MAPK by SpvC reduces expression of pro-inflammatory cytokines (e.g., IL-8, TNF $\alpha$ ) and diminishes inflammation and neutrophil infiltration at infection sites during early stages of infection. ${ }^{14,15}$ In addition to ERK inhibition, $S$. enterica employ the AvrA effector to block JNK signaling. AvrA is a SPI1 T3SS effector protein that is a close homologue of YopJ.46 Like YopJ, AvrA is an acetyl-transferase and under physiological conditions it acetylates specific serine residues of MKK4 and MKK7.16,17 The modified MAKK4/7 can no longer be phosphorylated and activated, thus specifically compromising JNK pathway, host inflammatory response and apoptosis. ${ }^{47}$ The $S$. enterica SteC effector is a serine/threonine kinase delivered through the SPI2 T3SS into the host cells by intercellular bacteria.18,19 SteC catalyzes MEK1 and MEK2 phosphorylation, triggering their activation by auto-phosphorylation, resulting in activation of ERK1/2 signaling. Through this activity, SteC promotes the formation of the F-actin meshwork around $S$. enterica-containing vacuoles. ${ }^{18,19}$ In conclusion, $S$. enterica exhibit intricate manipulation of MAPK signaling, particularly that of ERK1/2.

\section{Vibrio parahaemolyticus abol- ish the three main MAPK sig- naling cascades}

Vibrio parahaemolyticus (V. parahaemolyticus) is a Gram-negative halophilic bacterium inhabiting marine or estuarine environments. It is an important causative agent of gastroenteritis, usually related to the consumption of undercooked seafood.48 The $V$. parahaemolyticus T3SS effector VopA is an acetyl transferase, member of the YopJ acetyl-transferases protein family. Like YopJ, it modifies MKKs, including MKK1 and MKK6 on serine, threonine, and lysine residues. The VopA activity abolishes the three main MAPK signaling cascades, ERK, p38 and JNK, but does not affect $\mathrm{NF}-\kappa \mathrm{B} .{ }^{20}$ Interestingly, this virulence protein acetylates not only the activation loop of MKKs, but also a residue in the catalytic loop that is required for ATP binding, thereby blocking MAPKs in a dual way: i) inhibiting activation through phosphorylation; and ii) inhibiting the kinase function. ${ }^{21} \mathrm{An}$ additional effector of V. parahaemolyticus that down regulates MAPK and NF- $\mathrm{KB}$ cascades is VopZ, which leads to inhibition of MAPK through repression of TAK1 MAP3K. It is unclear whether VopZ acts directly on TAK1, and its enzymatic activity has yet to be uncovered.22

\section{Pseudomonas syringae tightly regulate the plant MAPK}

Pseudomonas syringae ( $P$. syringae) is a gram-negative plant pathogen that causes a wide variety of diseases, including blights, leaf spots, and galls in different plant species. ${ }^{49}$ To facilitate stable infection, $P$. syringae subvert MAPK signaling via T3SS effectors HopAI1 and HopF2 (Figure 2). HopAI1 belongs to the OspFlike protein family of phospho-threonine-lyases. In Arabidopsis, HopAI1 targets MPK3, MPK4 and MPK6 and inactivates them to suppress plant defense responses. ${ }^{24,25}$ An additional interesting $P$. syringae effector is HopF2, an ADP-ribosyltransferase that suppresses two branches of the MAPK cascade. Firstly, it catalyzes ADP-ribosylation of MKK5, thereby suppressing downstream MPK3 and MPK6 activation. ${ }^{23}$ In addition, it indirectly suppresses MPK4by inhibiting a component upstream of
MKK1, BAK1.50 Interestingly, BAK1 is also a target for additional $P$. syringae virulence factors, such as AvrPto and AvrPtoB. ${ }^{51}$

\section{Bacillus anthracis secrete a toxin to block MAPK signaling}

Bacillus anthracis (B. anthracis) the causative agent of anthrax, are Gram-positive, rod-shaped, spore-forming bacteria. A major virulence factor of this pathogen is the secreted anthrax toxin. The lethal factor subunit of this toxin is a zinc metalloprotease that cleaves the N-terminus from several MKKs, including MKK1-4 and MKK6-7, resulting in a kinase that can no longer interact with its substrate.26-28 The cleavage of these kinases leads to the inactivation of the ERK1/2, p38, and JNK signaling pathways. The inactivation of the MAPK pathway in macrophages and dendritic cells leads to inhibition of pro-inflammatory cytokine secretion, down regulation of costimulatory molecules such as CD80 and CD86, and ineffective $\mathrm{T}$ cell priming. The result is an impaired innate and adaptive immune response. ${ }^{52}$

\section{Mycobacterium tuberculosis changes the JNK phosphoryla- tion balance}

Nearly one-third of the world's population is a carrier of Mycobacterium tuberculosis (M. tuberculosis), the major causative agent of

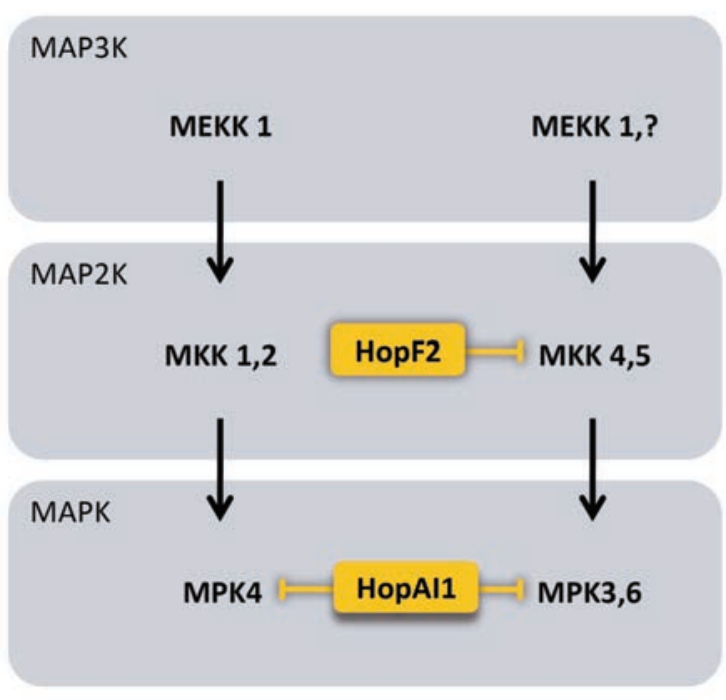

Figure 2. Manipulation of plant MAPK signaling by bacterial proteins. MAPK proteins are colored in black, and divided to three tiers: MAPK, MAP2K and MAP3K. Bacterial proteins that interfere with MAPK cascade are marked in squares. 
Table 1. Bacterial proteins that interfere with MAPK signaling.

\begin{tabular}{|c|c|c|c|c|c|}
\hline Microbe & $\begin{array}{l}\text { Bacterial effector/ } \\
\text { toxin }\end{array}$ & $\begin{array}{l}\text { Enzymatic } \\
\text { activity }\end{array}$ & $\begin{array}{l}\text { Host } \\
\text { substrate }\end{array}$ & $\begin{array}{l}\text { Subversion } \\
\text { mechanism* }\end{array}$ & References \\
\hline $\begin{array}{l}\text { Enteropathogenic } \\
\text { E. coli }\end{array}$ & $\mathrm{NleD}$ & Protease & p38, JNK & $\begin{array}{l}\text { Prevents p38/JNK } \\
\text { activation by cleaving } \\
\text { within their activation loops }\end{array}$ & 6 \\
\hline $\begin{array}{l}\text { Enteropathogenic } \\
\text { E. coli }\end{array}$ & NleE & $\begin{array}{l}\text { Methyl } \\
\text { transferase }\end{array}$ & $\mathrm{TAB} 2 / 3$ & $\begin{array}{l}\text { Blocks TAK1 activation by methylation } \\
\text { of the TAB2/3 } \\
\text { components of the TAK1 complex }\end{array}$ & 7 \\
\hline S. flexneri & OspF & $\begin{array}{l}\text { Phosphothreonine } \\
\text { lyase }\end{array}$ & ERK, p38 & $\begin{array}{l}\text { Irreversibly inactivate ERK and p38 } \\
\text { by cleavage of the C-OP } \\
\text { bond in the phosphothreonine } \\
\text { residue in the TXY motif }\end{array}$ & 8-10 \\
\hline Yersinia st. & YopJ & Acetyl transferase & $\begin{array}{c}\text { TAK1, } \\
\text { MKK2,4,6,7 }\end{array}$ & $\begin{array}{l}\text { Prevents MKK2,4,6,7 and TAK1 } \\
\text { phosphorylation by acetylating } \\
\text { residues in their activation loops }\end{array}$ & $11-13$ \\
\hline S. enterica & SpvC & Phosphothreonine lyase & ERK & $\begin{array}{l}\text { Similar to OspF } \\
\text { with preference to ERK } \\
\text { OspF homolog }\end{array}$ & $8,14,15$ \\
\hline S. enterica & AvrA & Acetyl transferase & MKK $4 / 7$ & $\begin{array}{l}\text { Similar to YopJ } \\
\text { Prevents MKK4 and MKK7 } \\
\text { phosphorylation by acetylating } \\
\text { residues in their activation loops }\end{array}$ & 16,17 \\
\hline S. enterica & SteC & Kinase & MEK1 & $\begin{array}{l}\text { Induce MEK1 and MEK2 activation } \\
\text { by phosphorylating them to promote } \\
\text { their auto-phosphorylation }\end{array}$ & 18,19 \\
\hline V. parahaemolyticus & VopA & Acetyl transferase & MKK1,6 & $\begin{array}{l}\text { Similar to YopJ } \\
\text { Prevents MKK1, } 6 \text { phosphorylation } \\
\text { by acetylating residues in their } \\
\text { activation loops and catalytic loops }\end{array}$ & 20,21 \\
\hline V. parahaemolyticus & VopZ & Unknown & Unknown & Inhibits activation of TAK1 & 22 \\
\hline P. syringae & HopF2 & $\begin{array}{l}\text { ADP-ribosyl } \\
\text { transferase }\end{array}$ & Plant MKK5 & $\begin{array}{l}\text { Blocks MKK5 kinase activity } \\
\text { by ADP-ribosylating it }\end{array}$ & 23 \\
\hline P. syringae & HopAIl & Phosphothreonine lyase & $\begin{array}{l}\text { Plant MAPK } \\
(\text { MPK3,4,6) }\end{array}$ & $\begin{array}{l}\text { Similar to OspF } \\
\text { Irreversibly removes the } \\
\text { phosphate moiety from a } \\
\text { phosphor-threonine } \\
\text { residue in the TXY motif } \\
\text { of the plant MPK3,4 and } 6\end{array}$ & 24,25 \\
\hline B. anthracis & LF & Protease & KK1-4, MKK6-7 & $\begin{array}{l}\text { Cleaves the N-terminus } \\
\text { from MKK1-4 and MKK6-7, } \\
\text { resulting in a kinase that cannot } \\
\text { interact with its substrate }\end{array}$ & $26-28$ \\
\hline M. tuberculosis & Eis & Acetyl transferase & DUSP16 & $\begin{array}{l}\text { Activates through acetylation the JNK } \\
\text { phosphatase DUSP16s } \\
\text { JNK is therefore inhibited by increased } \\
\text { dephosphorylation }\end{array}$ & 29,20 \\
\hline
\end{tabular}

*A more extensive description of the subversion mechanism.

tuberculosis, and the leading cause of death from a bacterial infection worldwide. 53 This pathogen invades and thrives in the host professional phagocytic cells, such as macrophages, neutrophils, monocytes and dendritic cells, by arresting phagosome maturation and fusion with lysosomes. $M$. tuberculosis secrete the enhanced intracellular survival (Eis) protein, an efficient $\mathrm{N}$-acetyltransferase that activates the JNK specific phosphatase DUSP16 through acetylation on Lys55. ${ }^{29}$ DUSPs are enzymes that can dephosphorylate, and thereby inactivate, both the threonine and tyrosine residues in the activa- tion loop of MAPKs. JNK is therefore inhibited by increased dephosphorylation, 30 which enhances pathogen survival inside cells by reducing autophagic cell death and inflammation. 54

\section{Closing remarks}

Microbes and hosts maintain an ongoing complex molecular cross talk. Bacteria, viruses and parasitic pathogens developed fascinating techniques to maneuver the host MAPKs sig- naling pathway and gain control over inflammation. Many bacteria have more than one effector that manipulates the MAPK pathway, differing in the MAPK substrates or time of action. Interestingly, some effectors block the MAPK cascade while others induce it, suggesting a complex balance between them. Although many bacterial effector mechanisms are being revealed in recent years, our understanding of how all the effectors cooperate in order to achieve a stable infection is still incomplete and represents a major challenge to future research. 


\section{References}

1. Ajibade A, Wang HY, Wang RF. Cell typespecific function of TAK1 in innate immune signaling. Trends Immunol 2013;34:307-16.

2. Banerjee A, Gerondakis S. Coordinating TLR-activated signaling pathways in cells of the immune system. Immunol Cell Biol 2007;85:420-4.

3. Baxt L, Garza-Mayers AC, Goldberg MB. Bacterial subversion of host innate immune pathways. Science 2013;340:697701.

4. Salomon D, Orth K. Lost after translation: Post-translational modifications by bacterial type III effectors. Curr Opin Microbiol 2013;16:213-20.

5. Raymond B, Young JC, Pallett M, et al. Subversion of trafficking, apoptosis, and innate immunity by type III secretion system effectors. Trends Microbiol 2013;21: 430-41.

6. Baruch K, Gur-Arie L, Nadler C, et al. Metalloprotease type III effectors that specifically cleave JNK and NF- $\kappa$ B. EMBO J 2011;30:221-31.

7. Zhang L, Ding X, Cui J, et al. Cysteine methylation disrupts ubiquitin-chain sensing in NF- $\kappa \mathrm{B}$ activation. Nature 2012;481:204-8.

8. Li H, Xu H, Zhou Y, et al. The phosphothreonine lyase activity of a bacterial type III effector family. Science 2007;315:1000-3.

9. Zhu Y, Li H, Long C, et al. Structural insights into the enzymatic mechanism of the pathogenic MAPK phosphothreonine lyase. Mol Cell 2007;28:899-913.

10. Arbibe L, Kim DW, Batsche E, et al. An injected bacterial effector targets chromatin access for transcription factor NFkappaB to alter transcription of host genes involved in immune responses. Nat Immunol 2007;8:47-56.

11. Mukherjee S, Keitany G, Li Y, Orth K. Yersinia YopJ acetylates and inhibits kinase activation by blocking phosphorylation. Science 2006;(May):1211-4.

12. Mittal R, Peak-Chew S-Y, McMahon HT. Acetylation of MEK2 and I kappa B kinase (IKK) activation loop residues by YopJ inhibits signaling. Proc Natl Acad Sci U S A 2006;103:18574-9.

13. Paquette N, Conlon J, Sweet C, et al. Serine/threonine acetylation of TGF -activated kinase (TAK1) by Yersinia pestis YopJ inhibits innate immune signaling. Proc Natl Acad Sci. 2012;109:12710-5.

14. Mazurkiewicz P, Thomas J, Thompson J, et al. SpvC is a Salmonella effector with phosphothreonine lyase activity on host mitogen-activated protein kinases. Mol Microbiol 2008;67:1371-83.
15. Haneda T, Ishii Y, Shimizu H, et al. Salmonella type III effector SpvC, a phosphothreonine lyase, contributes to reduction in inflammatory response during intestinal phase of infection. Cell Microbiol 2012;14:485-99.

16. Jones $\mathrm{RM}, \mathrm{Wu} \mathrm{H}$, Wentworth $\mathrm{C}$, et al. Salmonella AvrA coordinates suppression of host immune and apoptotic defenses via JNK pathway blockade. Cell Host Microbe 2008;3:233-44.

17. Du F, Galán JE. Selective inhibition of type III secretion activated signaling by the Salmonella effector AvrA. PLoS Pathog 2009;5:1-12.

18. Poh J, Odendall C, Spanos A, et al. SteC is a Salmonella kinase required for SPI-2dependent F-actin remodelling. Cell Microbiol 2008;10:20-30.

19. Odendall C, Rolhion N, Förster A, et al. The salmonella kinase SteC targets the MAP kinase MEK to regulate the host actin cytoskeleton. Cell Host Microbe 2012;12:657-68.

20. Trosky JE, Mukherjee S, Burdette DL, et al. Inhibition of MAPK signaling pathways by VopA from Vibrio parahaemolyticus. J Biol Chem 2004;279:51953-7.

21. Trosky JE, Li Y, Mukherjee S, et al. VopA inhibits ATP binding by acetylating the catalytic loop of MAPK kinases. J Biol Chem 2007;282:34299-305.

22. Zhou X, Gewurz BE, Ritchie JM, et al. A Vibrio parahaemolyticus T3SS effector mediates pathogenesis by independently enabling intestinal colonization and inhibiting TAK1 activation. Cell Rep 2013;3:1690-702.

23. Wang Y, Li J, Hou S, et al. A Pseudomonas syringae ADP-ribosyltransferase inhibits Arabidopsis mitogen-activated protein kinase kinases. Plant Cell 2010;22:2033-44.

24. Zhang J, Shao F, Li Y, et al. A Pseudomonas syringae effector inactivates MAPKs to suppress PAMP-induced immunity in plants. Cell Host Microbe 2007;1:175-85.

25. Zhang Z, Wu Y, Gao M, et al. Disruption of PAMP-induced MAP kinase cascade by a pseudomonas syringae effector activates plant immunity mediated by the NB-LRR protein SUMM2. Cell Host Microbe 2012;11:253-63.

26. Duesbery NS, Webb CP, Leppla SH, et al. Proteolytic inactivation of MAP-kinasekinase by anthrax lethal factor. Science 1998;280:734-7.

27. Pellizzari R, Guidi-Rontani C, Vitale G, et al. Anthrax lethal factor cleaves MKK3 in macrophages and inhibits the LPS/IFNgamma-induced release of NO and TNFalpha. FEBS Lett 1999;462:199-204.

28. Vitale G, Pellizzari R, Recchi C, et al. Anthrax lethal factor cleaves the N-terminus of MAPKKs and induces tyrosine/thre- onine phosphorylation of MAPKs in cultured macrophages. Biochem Biophys Res Commun 1998;248:706-11.

29. Masuda K, Shima H, Watanabe M, Kikuchi K. MKP-7, a novel mitogen-activated protein kinase phosphatase, functions as a shuttle protein. J Biol Chem 2001;276 :39002-11.

30. Kim KH, An DR, Song J, et al. Mycobacterium tuberculosis Eis protein initiates suppression of host immune responses by acetylation of DUSP16/MKP7. Proc Natl Acad Sci 2012;109:7729-34.

31. Lanata CF, Fischer-Walker CL, Olascoaga $\mathrm{AC}$, et al. Global causes of diarrheal disease mortality in children $<5$ years of age: a systematic review. PLoS One 2013;8: e72788.

32. Welinder-Olsson C, Kaijser B. Enterohemorrhagic Escherichia coli (EHEC). Scand J Infect Dis 2005;37:405-16.

33. Nadler C, Baruch K, Kobi S, et al. The type III secretion effector NleE inhibits NF-KB activation. PLoS Pathog 2010;6:e1000743.

34. Vossenkämper A, Marchès 0 , Fairclough $\mathrm{PD}$, et al. Inhibition of NF- $\mathrm{KB}$ signaling in human dendritic cells by the enteropathogenic Escherichia coli effector protein NleE. J Immunol 2010;185:4118-27.

35. Newton HJ, Pearson JS, Badea L, et al. The type III effectors NieE and NleB from enteropathogenic E. coli and Ospz from shigella block nuclear translocation of NFкB p65. PLoS Pathog 2010;6:1-16.

36. Mills E, Baruch K, Aviv G, Nitzan M. Dynamics of the type III secretion system activity of enteropathogenic Escherichia coli. MBio 2013;4:1-9.

37. Von Seidlein L, Deok RK, Ali M, et al. A multicentre study of Shigella diarrhoea in six Asian countries: Disease burden, clinical manifestations, and microbiology. PLoS Med 2006;3:1556-69.

38. Harouz H, Rachez C, Meijer BM, et al. Shigella flexneri targets the HP 1 c subcode through the phosphothreonine lyase OspF. EMBO J 2014;33:2606-22.

39. Zhou H, Monack DM, Kayagaki N, et al. Yersinia virulence factor YopJ acts as a deubiquitinase to inhibit NF-kappa B activation. J Exp Med 2005;202:1327-32.

40. Sweet CR, Conlon J, Golenbock DT, et al. YopJ targets TRAF proteins to inhibit TLRmediated NF-кB, MAPK and IRF3 signal transduction. Cell Microbiol 2007;9:2700-15.

41. Orth K. Function of the Yersinia effector YopJ. Curr Opin Microbiol 2002;5:38-43.

42. Keestra M, Winter MG, Klein-douwel D, et al. A Salmonella virulence factor activates the NOD1/NOD2 signaling pathway. MBio 2011;2:1-10.

43. Pilar AVC, Reid-Yu S, Cooper C, et al. GogB is an anti-inflammatory effector that limits tissue damage during Salmonella infec- 
tion through interaction with human FBX022 and Skp1. PLoS Pathog 2012;8:e1002773.

44. Steele-Mortimer 0, Brumell JH, Knodler L, et al. The invasion-associated type III secretion system of Salmonella enterica serovar Typhimurium is necessary for intracellular proliferation and vacuole biogenesis in epithelial cells. Cell Microbiol 2002;4:43-54.

45. Matsui H, Bacot CM, Doyle TJ, et al. Virulence plasmid-borne. Microbiology 2001;183:4652-8.

46. Mukherjee S, Hao YH, Orth K. A newly discovered post-translational modification the acetylation of serine and threonine residues. Trends Biochem Sci
2007;32:210-6.

47. Wu H, Jones RM, Neish AS. The Salmonella effector AvrA mediates bacterial intracellular survival during infection in vivo. Cell Microbiol 2012;14:28-39.

48. Su YC, Liu C. Vibrio parahaemolyticus: a concern of seafood safety. Food Microbiol 2007;24:549-58.

49. Preston GM. Pseudomonas syringae pv. tomato: the right pathogen, of the right plant, at the right time. Mol Plant Pathol 2000;1:263-75.

50. Zhou J, Wu S, Chen X, et al. Pseudomonas syringae effector HopF2 suppresses arabidopsis immunity by targeting BAK1. Changes 2012;29:997-1003.

51. Shan L, He P, Li J, et al. Bacterial effectors target the common signaling partner BAK1 to Disrupt multiple MAMP receptor-signaling complexes and impede plant immunity. Cell Host Microbe 2008;4:17-27.

52. Agrawal A, Pulendran B. Anthrax lethal toxin: a weapon of multisystem destruction. Cell Mol Life Sci 2004;61:2859-65.

53. Dye C, Williams BG. The population dynamics and control of tuberculosis. Science 2010;328:856-61.

54. Shin DM, Jeon BY, Lee HM, et al. Mycobacterium tuberculosis eis regulates autophagy, inflammation, and cell death through redox-dependent signaling. PLoS Pathog 2010;6:e1001230. 